\title{
Research on Correlation Analysis between Process Parameters of NC Machining and Quality Data Based on Grey Relational Analysis
}

\author{
Ce Wang ${ }^{1, a}$, Guijiang Duan ${ }^{1}$, Rui Liu ${ }^{1}$ and Xiong Han $^{2}$ \\ ${ }^{1}$ School of Mechanical Engineering and Automation, Beihang University, Beijing, 100191, China \\ ${ }^{2}$ AVIC Chengdu Aircraft Industrial (Group) Co.,Ltd, Chengdu, 610091, China
}

\begin{abstract}
With the wide use of CNC machine tools, data resources are gradually enriched. How to use the collected data resources for quality control becomes a hot topic for researchers. This paper introduces the use of grey correlation analysis theory to establish the relationship between the process parameters of CNC machine tools and quality data, and then provides a theoretical basis for the quality control using the process parameters of $\mathrm{CNC}$ machine tools.
\end{abstract}

\section{Introduction}

The integration of the new generation of information technology and manufacturing based on the internet has become an important feature and trend of the global intelligent manufacturing. The extensive use of advanced manufacturing technology and CNC machine tools provides the possibility for the acquisition, transmission and processing of manufacturing process information. How to correlate the acquired data resources with the quality of the quality of products under modern manufacturing mode, and timely according to the collected data resources, early warning of product quality and continuous improvement of process parameters that affect product quality have become one of the research hotspots in modern quality control.

Some companies have carried out relevant exploratory work on quality data acquisition, management, and analysis. However, there are still some certain problems to solve. Specifically, after the completion of data collection, there are some data islands, lack of effective analysis methods, and the inability to use their data values. Most researchers focus their attention on the quality control of the quality data itself. Few researchers consider the significance of process parameters of CNC machine tools for quality control. ${ }^{[1]}$

To solve the above problems, this paper establishes the mapping relationship and explores the relationship between the process parameters of CNC machine tools and quality data, and judges the status of the production process through the process parameters of $\mathrm{CNC}$ machine tools to achieve early warning of product quality.

\section{Grey correlation analysis summary}

\subsection{Basic ideas}

The grey relational analysis (GRA) is a method that uses grey relational order (grey relational order, GRO) to describe the strength, size, and order of relationships among factors. ${ }^{[2]}$ The basic idea is to use mathematical methods to study the geometric correspondence between factors based on the data of the factors. GRA is actually a quantitative analysis of dynamic indicators, which fully reflects the dynamic significance. GRA has been widely used in agriculture, industry, economy, management and other disciplines, and has achieved remarkable results. The technical connotation of grey relational analysis is: obtaining the difference information of the sequence time, establishing the difference information space, establishing the comparative measure of the calculated difference information and establishing the sequence relationship between the factors.

\subsection{Related Calculations}

Assume sequence

$$
\begin{aligned}
& X_{i}=\left(x_{i}(1), x_{i}(2), \ldots, x_{i}(n)\right) \\
& \mathrm{Y}_{j}=\left(y_{j}(1), y_{j}(2), \ldots, y_{j}(n)\right)
\end{aligned}
$$

The (1) and (2) start zeroing images are:

$X_{i}^{0}=\left(x_{i}^{0}(1), x_{i}^{0}(2), \ldots, x_{i}^{0}(n)\right)$

$Y_{j}^{0}=\left(y_{j}^{0}(1), y_{j}^{0}(2), \ldots, y_{j}^{0}(n)\right)$

Among (3) and (4), $x_{i}^{0}(k)=x_{i}(k)-x_{i}(1)$ and $y_{j}^{0}(k)=y_{j}(k)-y_{j}(1), \mathrm{k}=1,2, \ldots, \mathrm{n}$. After the initial value, the zero point of the starting point are:

$$
X_{i}^{\prime 0}=\left(x_{i}^{\prime 0}(1), x_{i}^{\prime 0}(2), \ldots, x_{i}^{\prime 0}(n)\right)
$$




$$
Y_{j}^{\prime 0}=\left(y_{j}^{\prime 0}(1), y_{j}^{\prime 0}(2), \ldots, y_{j}^{\prime 0}(n)\right)
$$

Among (5) and (6), $x_{i}^{\prime 0}(k)=\frac{x_{i}(k)}{x_{i}(1)}$ and $y_{j}^{0}(k)=$ $\frac{y_{j}(k)}{y_{j}(1)}, \mathrm{k}=1,2, \ldots, \mathrm{n}$.

Grey Absolute Correlation Calculation

$$
\begin{aligned}
& \left|X_{S_{i}}\right|=\left|\sum_{k=2}^{n-1} x_{i}^{0}(k)+\frac{1}{2} x_{i}^{0}(n)\right| \\
& \left|Y_{S_{j}}\right|=\left|\sum_{k=2}^{n-1} y_{j}^{0}(k)+\frac{1}{2} y_{j}^{0}(n)\right| \\
& \left|Y_{S_{j}}-X_{S_{i}}\right|=\mid \sum_{k=2}^{n-1}\left(y_{j}^{0}(k)-x_{i}^{0}(k)\right)+\frac{1}{2}\left(y_{j}^{0}(n)-\right.
\end{aligned}
$$$$
\left.x_{i}^{0}(n)\right)
$$

According to (7), (8), and (9), the grey absolute correlation between $X_{i}$ and $Y_{j}$ is:

$$
\varepsilon_{i j}=\frac{1+\left|x_{s_{i}}\right|+\left|Y_{s_{j}}\right|}{1+\left|X_{S_{i}}\right|+\left|Y_{S_{j}}\right|+\left|Y_{S_{j}}-X_{S_{i}}\right|}
$$

Grey Relative Correlation calculation

$$
\begin{aligned}
& \left|X_{S_{i}}^{\prime}\right|=\left|\sum_{k=2}^{n-1} x_{i}^{\prime 0}(k)+\frac{1}{2} x_{i}^{\prime 0}(n)\right| \\
& \left|Y_{S_{j}}\right|=\left|\sum_{k=2}^{n-1} y_{j}^{\prime 0}(k)+\frac{1}{2} y_{j}^{\prime 0}(n)\right| \\
& \left|Y_{s_{j}}^{\prime}-X_{s_{i}}^{\prime}\right|=\mid \sum_{k=2}^{n-1}\left({y^{\prime}}_{j}^{0}(k)-x_{i}^{\prime 0}(k)\right)+ \\
& \frac{1}{2}\left({y^{\prime}}_{j}^{0}(n)-{x^{\prime}}_{i}^{0}(n)\right)
\end{aligned}
$$

According to (11), (12), and (13), the grey relative correlation between $X_{i}$ and $Y_{j}$ is:

$$
\gamma_{i j}=\frac{1+\left|X s_{s_{i}}\right|+\left|Y_{s_{j}}\right|}{1+\left|X_{s_{i}}\right|+\left|Y_{s_{j}}\right|+\left|Y_{s_{j}}-X \prime_{s_{i}}\right|}
$$

Grey Comprehensive Correlation Calculation

The grey comprehensive correlation between $X_{i}$ and $Y_{j}$ is (general $\theta=0.5$ ):

$$
\rho_{i j}=\theta \varepsilon_{i j}+(1-\theta) \gamma_{i j}
$$

The grey comprehensive correlation not only reflects the degree of similarity between $X_{i}$ and $Y_{j}$, but also shows the closeness of $X_{i}$ and $Y_{j}$ relative to the rate of change of the starting point. It is a quantitative index that comprehensively expresses of the close relationship between sequences.

After calculation, grey comprehensive correlation matrix can be obtained.

$$
\psi=\left(\rho_{i j}\right)_{s \times m}=\left(\begin{array}{cccc}
\rho_{11} & \rho_{12} & \ldots & \rho_{1 m} \\
\rho_{21} & \rho_{22} & \ldots & \rho_{2 m} \\
\ldots & \ldots & \ldots & \ldots \\
\rho_{s 1} & \rho_{s 2} & \ldots & \rho_{s m}
\end{array}\right)
$$

\section{Correlation analysis of process parameters of NC machining and quality data}

\subsection{Association analysis model process parameters of NC machining and quality data}

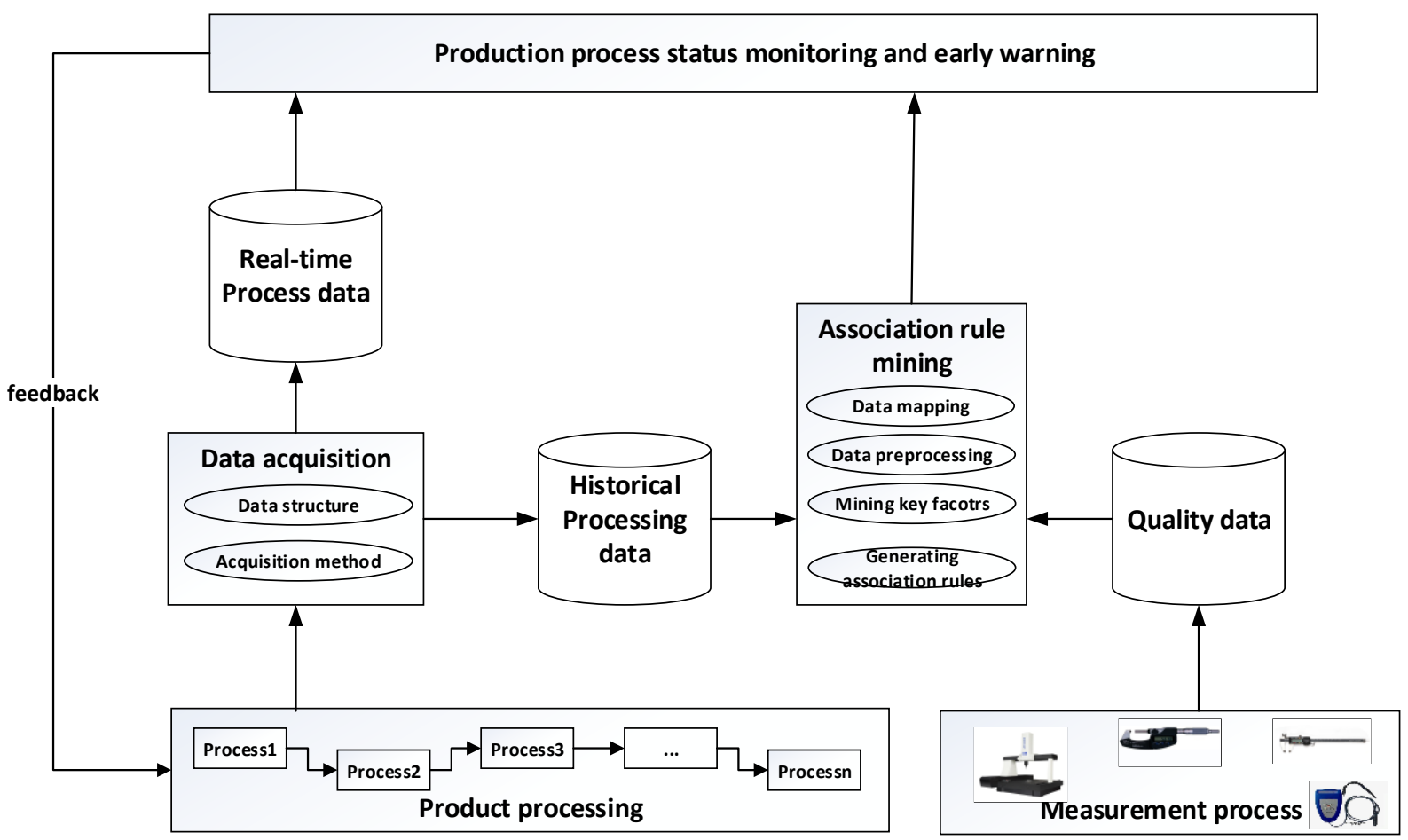

Figure 1. The process parameters of $\mathrm{CNC}$ machine tools and quality data association analysis model

As shown in the figure 1 , the correlation analysis model for process parameters of $\mathrm{CNC}$ machine tools and quality data is used to excavate the correlation rules between the process data and quality data to achieve effective monitoring of quality-impacted process parameters and ensure the stability of the production process and product quality. The model mainly includes the following modules $^{[3]}$ : (1) production process parameter data acquisition module, collects information such as spindle speed, spindle coordinate position and other numerical control processing equipment; (2) association rule mining module, uses the mapping relationship between process and processing, establishes the link between the process data and quality data, applying grey correlation analysis theory, mining key factors, generating association rules;(3) production process state monitoring and early warning, 
using real-time processing data of data collection and mining association rules to determine whether the production process is stable and feedback to the production line.

\subsection{Data acquisition}

The data acquisition of the processing process can be realized through the factory's DNC system. ${ }^{[4]}$ The DNC system of a certain factory can continuously collect the parameters such as spindle speed, coordinate position and current execution $\mathrm{NC}$ code of the $\mathrm{CNC}$ machine tools at the frequency of $10 \mathrm{~Hz}$, and record these parameters to the txt texts. To use these data, it need firstly to parse the txt texts and parse the data in the text into a set of parameters. In addition, it need to design a data structure that satisfies the subsequent association rule mining to store the data structure in the database for use by the association rule mining module. As shown in the table 1, the data structure of the machining process data includes not only the machining process parameter information such as the spindle speed and the feed speed, but also the time and the currently executed NC code and other tag information.
Table 1. The process parameters of CNC machine tools data structure

\begin{tabular}{|c|c|c|c|}
\hline Field & Meaning & Field & Meaning \\
\hline F_ID & $\begin{array}{l}\text { Figure } \\
\text { number }\end{array}$ & $\underset{\mathrm{e}}{\mathrm{NC} \_\mathrm{Cod}}$ & $\begin{array}{c}\mathrm{NC} \\
\text { program }\end{array}$ \\
\hline T_ID & Tool number & M_P & $\begin{array}{l}\text { Spindle } \\
\text { power }\end{array}$ \\
\hline M_I & $\begin{array}{l}\text { Spindle } \\
\text { current }\end{array}$ & M_S & $\begin{array}{c}\text { Spindle } \\
\text { speed }\end{array}$ \\
\hline F_Rate & Feed rate & F_Actual & $\begin{array}{l}\text { Actual } \\
\text { feed }\end{array}$ \\
\hline Cur_Code & $\begin{array}{l}\text { Current } \\
\text { segment }\end{array}$ & $\mathrm{X}$ & $\begin{array}{c}\text { Coordinat } \\
\text { e X }\end{array}$ \\
\hline $\mathrm{Y}$ & $\begin{array}{c}\text { Coordinate } \\
\text { Y }\end{array}$ & $\mathrm{Z}$ & $\begin{array}{c}\text { Coordinat } \\
\text { e Z }\end{array}$ \\
\hline A & $\begin{array}{c}\text { Coordinate } \\
\text { A }\end{array}$ & B & $\begin{array}{c}\text { Coordinat } \\
\text { e B }\end{array}$ \\
\hline T_Length & Tool length & Time_N & $\begin{array}{c}\text { Current } \\
\text { time }\end{array}$ \\
\hline
\end{tabular}

\subsection{Association rules mining}

Association rules mining includes the following steps: data mapping, data preprocessing, mining key factors, and generating association rules. ${ }^{[5]}$

(1)Data mapping: The process data and quality data do not have a direct mapping relationship, but the parts are processed by the CNC machine tools in a certain time period to execute a specific NC program to process each feature, and the feature generates quality data through detection. Therefore, the process data and quality data have been linked with the NC program through time.

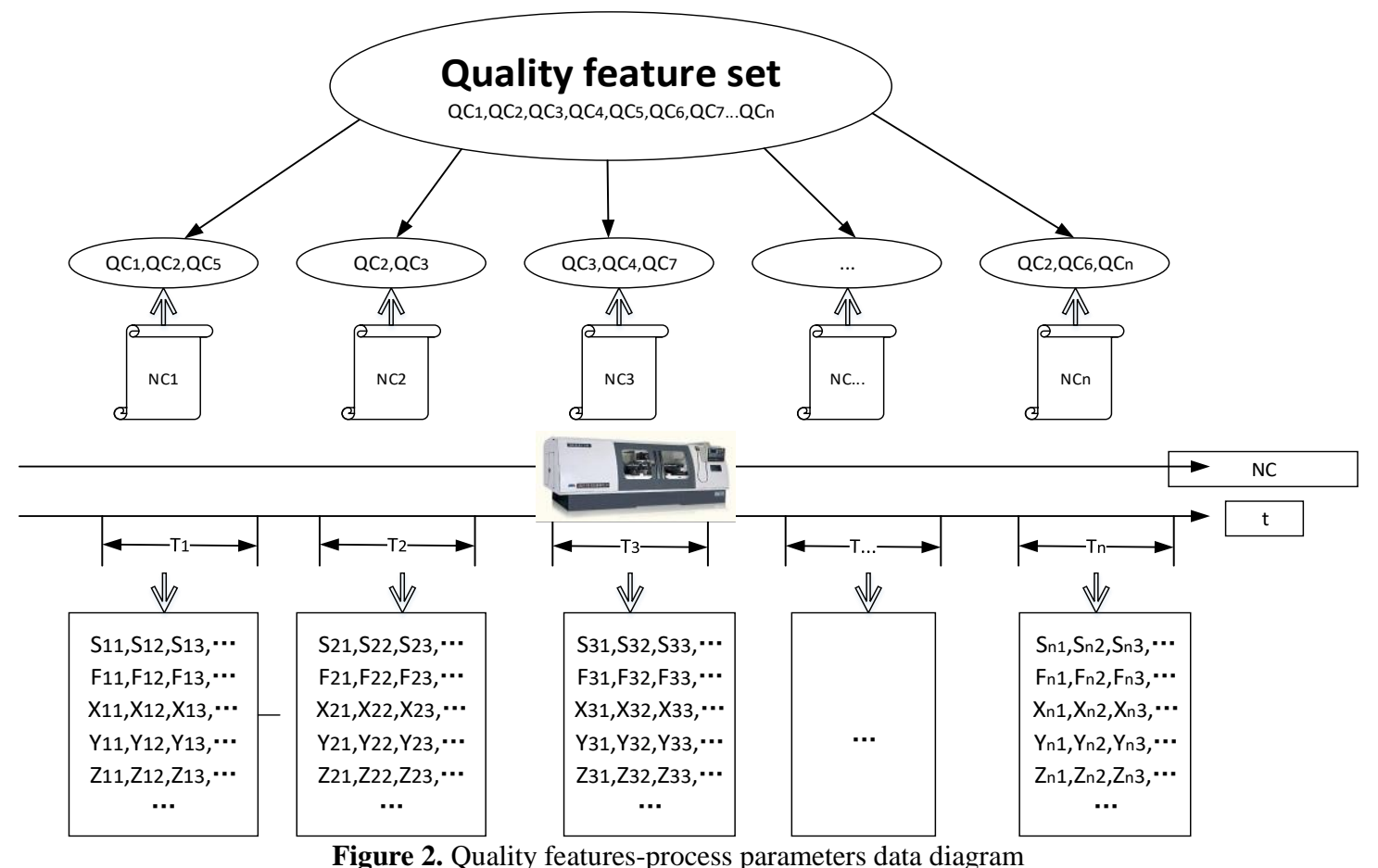

As shown in the figure 2 , the process data-time-NC block-feature-quality data has a correspondence relationship. The process data and quality data pass through the time-NC block-feature. The bridge establishes a mapping relationship.
(2)Data preprocessing: The frequency of data acquisition is $10 \mathrm{~Hz}$. Although the same NC program is executed, the time spent is not necessarily the same. Therefore, the amount of data is not the same. It is not easy to discover the correlation rules by using the original 
data. The statistical features of the data are more concise and easy to find the correlation rules. The statistical features of a group of data are mainly described in terms of trend, degree of dispersion, and distribution morphology. Because of the different amount of data in the process, the features of the \{numeric mean, standard deviation, skewness $\}$ characterization data are selected.

$$
\left\{\bar{x}=\frac{\sum_{i=1}^{\mathrm{n}} x_{i}}{n}, \sigma=\sqrt{\frac{\sum_{i=1}^{\mathrm{n}}\left(x_{i}-\bar{x}\right)^{2}}{n}}, S k=\frac{1}{n-1} \sum_{i=1}^{n}\left(x_{i}-\right.\right.
$$

$\left.\bar{x})^{3} / \sigma^{3}\right\}$

(3)Mining key factors: The parameters of NC machining process can be expressed as $S\{\bar{x}, \sigma, S k\}$ using the spindle speed $\mathrm{S}$ as an example, then the parameters of the $\mathrm{NC}$ machining process can be expressed as (18).

$$
N C P=\{S 1\{\bar{x}, \sigma, S k\}, S 2\{\bar{x}, \sigma, S k\},
$$

$\cdots, F 1\{\bar{x}, \sigma, S k\}, F 2\{\bar{x}, \sigma, S k\}, \cdots, \cdots\}$ The quality feature set is $\mathrm{QC}=$ $\{Q C 1, \quad Q C 2, \quad \cdots\}$, use the grey correlation analysis in Chapter 2 to obtain:

$$
\psi=\left(\rho_{i j}\right)_{s \times m}=\left(\begin{array}{cccc}
\rho_{11} & \rho_{12} & \cdots & \rho_{1 m} \\
\rho_{21} & \rho_{22} & \cdots & \rho_{2 m} \\
\cdots & \ldots & \cdots & \ldots \\
\rho_{s 1} & \rho_{s 2} & \cdots & \rho_{s m}
\end{array}\right)
$$

For each row $\rho$ seeking maximum value, suppose $\rho_{12}$ in the first row is the maximum value, then QC1 has the highest degree of correlation with $\mathrm{S} 2$, and $\mathrm{S} 2$ is the key factor in QC1 processing.

(4)Generating association rules: use mathematical statistics to determine the sign of $\rho^{[6]}$, then:

$\mathrm{QC} 1 \propto \rho_{11} \times S 1+\rho_{12} \times S 2-\rho_{13} \times F 1+\cdots+$ $\rho_{1 m} \operatorname{In}(20)$

\section{Conclusion}

There is a link between the process parameters of CNC machine tools and quality data. This paper establishes the mapping relationship between the process parameters of $\mathrm{CNC}$ machine tools and quality data through the time-NC block-features, and uses the grey correlation analysis theory to construct the correlation between the process parameters of CNC machine tools and quality data. Through the relationship between the process parameters of CNC machine tools and quality data, the state of the production process can be judged through the process parameters of CNC machine tools to achieve product quality early warning.

\section{Acknowledgement}

This work was supported by the Graduate Student Innovation Fund of Beihang University (YCSJ-03-201709) and AVIC Chengdu Aircraft Industrial (Group) Co.,Ltd. The authors would like express their appreciation to the agency.

\section{References}

1. Yihui L, Shudong S, etc Aeronautical Manufacturing Technology 2 28(2005)

2. Xuerui T, Julong D Statitical Research 6546 (1995)

3. Jinglei Q, Shaobo L, Jinkun C Computer Integrated Manufacturing Systems 23 1963(2017)

4. A Krenzel. Computer-aided data collection system for assisting in analyzing critical situations. US Patent 5,124,915, (1992)

5. Baowen X, Weifeng Z Chinese Journal of Computers. 4 430(2001)

6. Bo S, Lu L, Fangting Y System Engineering Theory and Practice 9 98(2008) 